\title{
Uncertainties in greenhouse gases inventories - expanding our perspective
}

\author{
Jean P. Ometto • Rostyslav Bun • Matthias Jonas • \\ Zbigniew Nahorski • Mykola I. Gusti
}

Received: 9 April 2014 / Accepted: 5 May 2014 / Published online: 24 May 2014

(C) Springer Science+Business Media Dordrecht 2014

Strategies for mitigating global climate change require accurate estimates of the emissions of greenhouse gases (GHGs). A strong consensus in the global scientific community states that efforts to control climate change require stabilization of the atmospheric concentration of GHGs (as per a recent compilation; (IPCC 2013)). Estimates of the amounts of carbon dioxide and other GHGs emitted to the atmosphere, as well as the amounts absorbed by terrestrial and aquatic systems, are crucial for planning, analyzing, validating and at global scale verifying mitigation efforts and for analyzing scenarios of future emissions. The magnitude and distribution of current emissions and the path of future emissions are both of considerable importance. It is critical that we have estimates of emissions and that we acknowledge and deal with the uncertainty in our best estimates. The range of issues that derive from uncertainty in emissions estimates was the subject of the $3^{\text {rd }}$ International Uncertainty Workshop held in Lviv, Ukraine, 2010, and is the subject of this special issue.

Resolving national or regional contributions to changes in atmospheric GHG concentrations involves international agreements and national inventories of emissions. Countries, cities, companies, and individuals are now commonly calculating their GHG emissions, and markets

This article is part of a Special Issue on "Third International Workshop on Uncertainty in Greenhouse Gas Inventories" edited by Jean Ometto and Rostyslav Bun.

J. P. Ometto $(\bowtie)$

Earth System Science Centre, National Institute for Space Research (CCST/INPE), Av dos Astronautas, 1758, 12227-010 São José dos Campos, SP, Brazil

e-mail: jean.ometto@inpe.br

R. Bun

Department of Applied Mathematics, Lviv Polytechnic National University, P.O.Box 5446, Lviv 79031, Ukraine

M. Jonas · M. I. Gusti

Advance Systems Analysis Program, International Institute for Applied Systems Analysis (IIASA),

Schlossplatz 1, A 2361 Laxenburg, Austria

Z. Nahorski

Systems Research Institute, Polish Academy of Sciences, Newelska 6, 01-447 Warsaw, Poland

M. I. Gusti

Department of International Information, Lviv Polytechnic National University, 1 Sviatoho Yura Square, Lviv 79031, Ukraine 
exist that allow trading emissions permits of carbon. Companies report corporate-level emissions or even the carbon footprint of products. But GHG emissions are seldom measured directly. For instance, it may be considered important that total, and trend, uncertainty in national emissions estimates is smaller than the reductions to which countries agree to under an international compliance regime, as well that emissions mitigation strategies, and trade, be based on accurate knowledge of the magnitudes and sources of emissions.

The 2010 United Nations Framework Convention on Climate Change (UNFCCC-COP 16; Cancun, Mexico) produced an agreement with the desire to limit global average surface temperature to $2^{\circ} \mathrm{C}$ above the pre-industrial level. To achieve this objective, the total amount of greenhouse gas emissions emitted to the atmosphere in 2020 has to be targeted at around 44 $\mathrm{Pg} \mathrm{CO}_{2}$-eq, from the current estimated value of $48 \mathrm{Pg} \mathrm{CO}_{2}$-eq [assuming a linear target path]. However the current emissions trajectories follow the most carbon intensive path of the recently published scenarios of the Intergovernmental Panel on Climate Change Fifth Assessment Report (IPCC 2013) (based on Representative Concentration Pathways; www. globalcarbonproject.org/). Experience with the Kyoto Protocol shows that quantitative estimation of uncertainty increases the value of the inventory provided by reporting authorities. Yet, only a few Annex I Parties report full uncertainty analysis, although default methods and underlying data are available for all countries.

UNFCCC reporting should be improved as Parties report comprehensive uncertainty analysis of the GHG inventory estimates and provide validation reports (for data and models used). The Intergovernmental Panel on Climate Change (IPCC) has proposed standardized methodologies for adequate accounting of national, natural and human-induced GHG sources and sinks. The methods, applied to national scales, have guided the production of emissions assessments at the country level for several years. Comparable methodologies have been developed within countries and trade groups. The constant evolution of the IPCC scientific review, associated with increasing international concerns over anticipated changes in the future climate, has raised a number of issues about compliance and verification, and about proposed and agreed strategies meant to reduce the impact on the global climate associated with human activities.

Because of the accumulation of GHGs in the atmosphere, concern focuses on not just current rates of emissions but on the trend in emissions and in cumulative emissions totals. Cumulative GHG emission budgets (i.e., for 2000-2050) have been shown to be a robust indicator for global temperatures at, and beyond, 2050 (Meinshausen et al., 2009), and are thus well suited to link long-term global warming targets with near and mid-term emissions. Cumulative global emissions targets can be translated into near term national emissions objectives, but uncertainty in both natural and anthropogenic fluxes of GHGs must be incorporated in monitoring and projecting emissions trends.

The international workshop in Lviv, Ukraine, was the third in a series exploring the magnitude and implications of uncertainty in GHG emissions estimates. Papers presented at the workshop and peer reviewed for this Climatic Change Special Issue explore the uncertainty in emissions estimates but also focus on detecting and evaluating changes in emissions; independent monitoring and verification of emissions estimates; and determining how to obtain critical information, and how to proceed without information that cannot be obtained. The papers are presented under general themes such as: Spatial Inventories; Land Use, Land Use Change and Forestry; Energy; Non- $\mathrm{CO}_{2}$ and Waste emissions; Economy and Climate Change; and General \& Policy.

In General \& Policy, Jonas et al. ask how uncertainty over time will affect short-term GHG emission commitments and long-term efforts to meet global temperature targets for 2050 and beyond. The study addresses a fundamental problem: how to combine uncertainty about current and historic emissions (diagnostic uncertainty) with uncertainty about projected future emissions (prognostic uncertainty). Although the authors' mode of bridging uncertainty across 
temporal scales still relies on discrete points in time and is not yet continuous, their study takes a valuable first step toward that objective. The proposed emissions-temperature-uncertainty framework assumes that cumulative emissions can be constrained over time by binding international agreements, as well as that emissions can be estimated only imprecisely, and whether or not they will achieve an agreed temperature target is also uncertain. The framework allows policymakers to understand diagnostic and prognostic uncertainty so that they can make more informed (precautionary) decisions for reducing emissions given an agreed future temperature target. The paper by Rafaj et al. examines key factors that have driven the observed evolution of $\mathrm{SO}_{2}, \mathrm{NO}_{\mathrm{x}}$, and $\mathrm{CO}_{2}$ emissions in Europe from 1960 to 2010, contributing to the understanding of the relationship between emissions and economic growth. It has often been suggested that emissions first increase with growing income and social welfare and subsequently decrease once a certain level of wealth has been attained. However, the authors' analysis demonstrates that observed turning points occur for different countries and pollutants at different income levels, and no turning point has yet been identified for $\mathrm{CO}_{2}$. Although there are factors determined by economic parameters (e.g. energy intensity, fuel mix, technological advances), the results provide little evidence that the emission control measures are directly linked to economic growth, but their adoption is rather driven by enforcement of deliberate mitigation policies. The methodology presented by the authors provides a quantitative basis for investigating uncertainties related to the determinants of emission projections. Their exemplary decomposition analysis allows for identifying those parameters that are most relevant in assessing the uncertainty of GHG emission inventories. Under the same theme, Lesiv et al. deal with the change in the uncertainty of emission estimates which, in general, results from both learning (improvement of knowledge) and the structural change in emissions (change in emitters). Understanding the change in uncertainty due to the two processes and being able to distinguish between them becomes particularly important under a compliance regime when countries claim fulfillment of their commitments to reduce or limit emissions, or for trading emission quotas under such a regime. In the first part of their study, focusing on the individual Member States of the former EU-15, the historical change in the total uncertainty of $\mathrm{CO}_{2}$ emissions from stationary sources is analyzed. In the second part of their study, the authors present examples of changes in total uncertainty considering scenarios of structural changes in the emitters consistent with the EU's "20-20-20" targets. This exercise shows that the increased knowledge of inventory processes has determined the change in total uncertainty in the past and should also be considered as the driving factor in the prospective future. In the final contribution within General \& Policy, Hryniewicz et al. return to the problem of checking compliance of uncertain GHG inventories with agreed emission targets. That is, why a direct comparison of emissions with targets is not scientifically robust. The starting point of this study is the IPPC Good Practice Guidelines statement that reporting of inventories should be consistent, comparable and transparent. Thus, there exists the need to explain why inventoried emissions satisfy a target or are closer to it in one case than in another. This idea led the authors to look at a compliance procedure via comparison of uncertain alternatives. Traditionally, probabilistic methods have been used, in which emissions are treated as a random variable. Comparison rules based on moments, such as mean values and variances, are not suitable for the comparison of emissions. More appropriate methods use percentiles and critical values, like a so-called undershooting technique which was discussed earlier, e.g. by Nahorski et al. (Nahorski et al. 2007) and Nahorski and Horabik (Nahorski and Horabik 2010). However, emissions are inventoried usually only once per year, and they are typically not random, so it is difficult to treat them as probabilistic variables. This is why possibility theory, which has grown out of the fuzzy sets, is more suitable to the problem. A possibility distribution is not based on frequencies of observations, but may be constructed, e.g., by experts. Despite the 
differences in the probability/possibility paradigms, the methods behind both approaches show similarities and the checking rules are often alike. Taking uncertainty into account, additional parameters are proposed to be included in a checking rule: how stringent do we understand compliance; or to which extent is the target met. Such a rule allows for classifying inventories: how credible are these in satisfying the target? This information can be used in elaborating advanced decision rules, which would allow for taking a more or less conservative position.

The evolution in reducing uncertainty in emissions estimates reflects: (1) improvements in knowledge within the scientific community (e.g., more precisely known emission factors and improvements in energy data); and (2) structural changes in the emissions (e.g., an increasing fraction of emissions from the sectors where data can be estimated with smaller uncertainty, such as energy). Within the Energy category of the contribution to this Special Issue volume, Uvarova et al. focus on a prime emitter - emissions from oil operations in the Russian Federation. The authors provide a good example to illustrate the impact of learning. They investigate improvement in accuracy of emission estimates under a shift of accounting methods: from the production-based IPCC (IPCC 2000) Tier 1 to the mass-balance-based IPCC (IPCC 2006) Tier 2. The authors' comparison shows that the estimates in accordance with the higher-tier method result in a greater accuracy and lower relative uncertainty (26\% under Tier 2 versus $54 \%$ under Tier 1). The authors suggest that this uncertainty can be reduced further, e.g., by improving the accuracy of the parameters, including the use of more geographically explicit emission factors, employed in the emissions calculations.

Furthermore, in the session dedicated to emissions associated with Land Use, Land Use Change and Forestry, Ometto et al., explore uncertainties associated with emissions related to land use change in the tropics, focusing on deforestation. As reported by Le Quéré et al. (Le Quéré et al. 2013), net emissions from deforestation are decreasing, although this issue is far from resolved. The carbon stock in the terrestrial biosphere is enormous and the pressure for land use and agricultural expansion is constant, especially in tropical systems (Dalla-Nora et al. 2014). The methods currently adopted to estimate the spatial variation of above- and belowground biomass in tropical forests are usually based on remote sensing analyses coupled with field datasets. Field measurements in tropical forests are, typically, relatively scarce and often limited in their spatial distribution. Thus, lack of data is one major step to be overcome concerning reducing uncertainty in estimating GHG emissions from land use change, in particular in tropical regions. In this paper, the authors do a comparative analysis of recently published biomass maps of the Amazon region, including the official data used by the Brazilian government for its report to the UNFCCC Secretariat. Among the outcomes of their analysis, the evolvement to higher resolved, spatially distributed forest biomass data is key to reduce uncertainty in emissions estimates in tropical regions. Establishing national systems of GHG emissions estimation and reporting in Land Use, Land Use Change, and Forestry (LULUCF) is under continuous improvement, with key features given by the availability of datasets and in-country improving capacity of data generation. However, regional harmonization of methods involved in national GHG estimation systems is rather poor. There are also necessary within-country steps toward better coordination of the research effort supporting GHG estimation, reporting and accounting under UNFCCC requirements. As well, the increase of data availability for external evaluation is an important step further toward better estimates of uncertainty.

The spatial distribution and estimates of emissions are further explored in the Spatial Inventories Section. Emission inventories with high spatial and temporal resolution can be related to a process-level understanding of emissions sources and yield many advantages in the realm of designing and evaluating emission control strategies; and they would be very helpful for climate models and for monitoring emissions and checking emissions commitments in 
greater detail where necessary. Boychuk and Bun, also referring to the Energy Section of this Special Issue, present a Geographical Information System (GIS) approach to the spatial inventory of GHG emissions in the energy sector. It includes the mathematical background for creating the spatial inventories of point-, line-, and area-type emission sources, caused by fossil-fuel use for power and heat production, the residential sector, industrial and agricultural sectors, and transport. The approach is based on the IPCC guidelines, official statistics on fuel consumption, and digital maps of the region under investigation. As an example, the western Ukraine region with an area of $110.6^{\text {th }} \mathrm{km}^{2}$ was used for experiments. The uncertainty of inventory results is calculated, and the results of sensitivity analysis are investigated. The approach proposes that allocating emissions to the places where they actually occur helps to improve the inventory process and to reduce the overall uncertainty. Such methodology is useful for large countries with uneven distribution of emission sources. Spatial inventories support decision making in reducing emissions at the regional level. Such mathematical tools and algorithms can also be used in climate models, for the analysis and prediction of the emission processes and their structure for a variety of scenarios. In a similar vein, Horabik and Nahorski present an original approach to allocating spatially correlated data, such as GHG emission inventories, to finer spatial scales, based on covariate information observable in a fine grid. This approach is useful for data disaggregation, like activity data in some categories of human activity, during GHG spatial inventory. Dependences are modeled with the conditional autoregressive structure introduced into a linear model as a random effect. The maximum likelihood approach to inference is employed, and the optimal predictors are developed to assess missing values in a fine grid. The authors propose a relevant disaggregation model and illustrate the approach using a real dataset of ammonia emission inventory in a region of Poland in $15 \mathrm{~km}, 10 \mathrm{~km}$, and $5 \mathrm{~km}$ grids. For the considered inventory, the fourfold allocation benefits greatly from the incorporation of the spatial component, while for the ninefold allocation, this advantage is limited, but still evident. Also, the proposed method is found to be particularly useful in correcting the prediction bias encountered for upper range emissions in the linear regression models. In this case study, the authors use the original data in a fine grid to assess the quality of resulting predictions, but for the purpose of potential applications, they also developed a relevant measure of prediction error. It is an important step to quantify the prediction error in situations, where original emissions in a fine grid are not known. The method of improving resolution opens the door to uncertainty reduction of spatially explicit GHG emission inventories.

Processing spatial data, such as GHG inventories, poses several problems, as the data are represented as grids. Verstraete proposes an approach to optimize the mapping of values in mismatched grids. When data that are represented using different grids need to be combined, the main problem is that the underlying distribution of activity data or any other parameter is not known, and thus a remapping from one grid onto another grid is difficult. Traditional methods work by making simple assumptions regarding the underlying distribution, but as those often do not match reality, it decreases the accuracy of the data. However, often there is knowledge available that can help with better estimating the real distribution. In the article, the author presents a new method, which allows additional data to be used. The method presented uses techniques from artificial intelligence (fuzzy sets, inference systems, etc.) to determine how one grid can be remapped onto another grid. Even with additional data, this is not straightforward, as data may not match exactly or may be incomplete. The article describes the concept of the approach, and discusses the results of experiments on artificial datasets.

Joerss, contributing to the Non- $\mathrm{CO}_{2} /$ Waste Section, compares results of air pollutant inventories from several European countries with the results of the PArticle REduction Strategies (PAREST) research project in Germany. The author uses a Monte-Carlo simulation 
for assessing the uncertainties in emissions of particulate matter (PM10 \& PM2.5) and aerosol precursors $\left(\mathrm{SO}_{2}, \mathrm{NO}_{\mathrm{x}}, \mathrm{NH}_{3}\right.$ and $\left.\mathrm{NMVOC}\right)$. The methodology and analysis for uncertainty assessment in the emissions inventories is successful for particulate matter and aerosol precursors. The uncertainty of the pollutant species analyses is determined and falls in the range of recent uncertainty assessments of European countries. The analysis by $X u$ et al, also part of this section, reveals a link between Non- $\mathrm{CO}_{2}$ emissions and economy, where the authors tackle the problem of uncertainty assessment in coal mine methane emissions projects, and estimation of its impact on a negotiated Certified Emission Reduction (CER) price. They use the Rubinstein-Ståhl bargaining model to fill the gaps in the database and to simulate negotiations concerning CER price, assuming that a buyer's willingness to negotiate a CER depends on the uncertainty associated with the emission reduction. The bargaining model is broadened by introducing dependence of some parameters on the probability of a contracted CER amount not to be realized. To quantify this probability, the authors develop a conditional distribution given information on the point estimate of methane emissions for the project under consideration, and on the distribution of available estimates from coalmines having similar characteristics. The proposed methodology is applied to a coalmine methane project implemented in the Huainan coalmine, the Anhui Province in Eastern China. The parameters of uncertainty distribution of the methane content are estimated using data, which are gathered from 25 Chinese coalmines with similar geological conditions. The results indicate that the uncertainty influence on price is significant, particularly when the credibility of a seller increases, and the probability of a failure to fulfill the project decreases.

The aggregated impact of climate change on society, economy and ecosystems, comprises the total impact across regions. Producing results of aggregated impact involves the challenge of discerning how adaptation will occur in society and ecosystems (what is the resilience of natural systems) and what are the paths that future development (economic and social) will follow (IPCC AR5). The section dedicated to the Economy \& Climate Change brings some of these elements to the discussion. Ermolieva et al. develop a novel trading-market model which mimics decentralized bilateral trade of emission permits under uncertainty. In contrast to existing emissions markets, the proposed model allows for addressing long-term socio-economic and environmental consequences of trade, irreversibility, and inherent uncertainty including asymmetric information of agents (countries). The model relies on an anonymous computerized optimization system (computerized market system) that can be viewed as "cloud computing". Trading between both countries and regions is shown to be robust. The trading process converges to the core solution and the trading parties create the stable (core) solution without incentives to leave the trading coalition. Numerical results show that the explicit treatment of uncertainty may significantly change the trading process by turning sellers of emission permits into buyers.

Looking at the carbon market under the Kyoto Protocol, Nahorski et al. present a simulation system that mimics trading of GHG emissions among parties, according to the Protocol's rules. It is admitted that the emissions are uncertain and this knowledge affects the trading rules, as presented in earlier studies by Nahorski et al. (Nahorski et al. 2007) and Nahorski and Horabik (Nahorski and Horabik 2010). These rules lead to more uncertain emissions that are less expensive on the market. The simulation does not assume an ideal market: the equilibrium prices are not known during the trade. Bilateral negotiations and sealed bid reverse auctions are considered for pricing the traded emissions. Only transactions profitable for both participants are accepted. A multi-agent approach is used as a tool for simulating the trading process. Nonlearning and learning agents are considered. The former use fixed probability distributions for placing orders, while the latter learn to modify the distribution according to the success/lack 
of success in winning transactions. Negotiation examples present phenomena similar to those spotted in real markets.

Following up on carbon trading, and on the influence of uncertainty on driving the market and defining prices, Dolgopolova et al. employ system dynamics models to analyze the impact of different uncertainties on emission trading - both on national and business levels. Economic, institutional and technological uncertainties determine the benefits from trading emissions permits. For any country participating in an international trading market, the uncertainty in the price range becomes crucial. In the case of business investment decisions for implementing resource-saving technologies, the proposed system dynamics model shows that the first-mover investor will obtain significantly fewer advantages than his followers, which leads to a delay in primary investments.

Nijnik \& Pajot analyze the social function of forests and the opportunity for mitigation through maintaining and replanting trees, and discuss the economic impact of dealing with uncertainties in using forests to mitigate climatic change. Limiting the analysis of uncertainty to discounting, the authors challenge the traditional cost-benefit analysis. Different settings of discounting are tested for carbon sequestration of the forestry sector in Scotland and Ukraine. The policy consequences of the exercise are also investigated. The choices of discounting protocols are shown to have a major influence on both the economic analysis and the decision-making process, which directly affect the climate change mitigation strategies in these countries. The authors highlight the implications on the policy decisions when uncertainty is considered in mitigating climate change through forestry.

Changes in relative uncertainty over time and scientific understanding of the main determinants of that change have obvious implications, e.g., for assessing the uncertainty of emissions with regard to compliance with emission reduction commitments and for trading emission quotas under the Kyoto Protocol or REDD+ mechanisms. Advances in methodology and mathematical modelling to constrain uncertainties associated with ecosystems and some carbon pools, are observed. However, investments in methodology-oriented research are particularly important for a full-system uncertainty estimate. In terrestrial systems, historical patterns and long-term datasets are important to draw a more accurate picture of the carbon pools evolution. In this respect we see the following scientific advances evolving from the workshop that should be considered in future studies: (i) combining diagnostic and prognostic uncertainty in a (e.g.) emissions-temperature setting that seeks to constrain global warming and linking uncertainty consistently across temporal scales; (ii) developing methodologies and information technologies that allow estimating GHG emissions and sinks with lower uncertainties, e.g., spatial GHG cadastres, and higher level tier methods; (iii) evaluating the influence of uncertainty on GHG emission markets aiming at robust and efficient emission trading; (iv) studying issues that influence the dynamics of GHG emissions estimates, e.g., learning curves and structural changes in emitters, as well as social, political and economic drivers, etc.; (v) constraining uncertainties in land use change emissions, as having great potential for reduction, and per its influence on ecosystem services and social aspects; and developing marked strategies for making emissions reduction economically attractive.

\section{References}

Dalla-Nora EL, de Aguiar APD, Lapola DM et al. (2014) Why have land use change models for the Amazon failed to capture the amount of deforestation over the last decade? Land Use Policy (http://dx.doi.org/10. 1016/j.landusepol.2014.02.004). 
IPCC (2000) Good practice guidance and uncertainty management in national greenhouse gas inventories. IPCC National Greenhouse Gas Inventories Programme. IGES/OECD/IEA, 2000

IPCC (2006) In: Eggleston HS, Buendia L, Miwa K, Ngara T, Tanabe K (eds) 2006 IPCC Guidelines for National Greenhouse Gas Inventories, Prepared by the National Greenhouse Gas Inventories Programme. IGES, Japan

IPCC (2013) The Physical Science Basis. Contribution of Working Group I to the Fifth Assessment Report of the Intergovernmental Panel on Climate Change [Stocker TF, Qin D, Plattner GK, Tignor M, Allen SK, Boschung J, Nauels A, Xia Y, Bex V and Midgley PM (eds.)]. Cambridge University Press, Cambridge, United Kingdom and New York, NY, USA, 1535 pp

Le Quéré C, Peters GP, Andres RJ (2013) Global carbon budget 2013. Earth Syst Sci Data Discus 6:689-760. doi:10.5194/essdd-6-689-2013

Nahorski Z, Horabik J (2010) Compliance and emission trading rules for asymmetric emission uncertainty estimates. Clim Chang 103(1-2):303-325

Nahorski Z, Horabik J, Jonas M (2007) Compliance and emission trading under the Kyoto Protocol: Rules for uncertain inventories. Water Air Soil Pollut Focus 7(4-5):539-558 